\author{
Yangqiu $\mathrm{Hu}^{1}$ \\ Department of Bioengineering, \\ University of Washington, \\ Seattle, WA 98195: \\ Department of Radiology, \\ University of Washington, \\ Seattle, WA 98195
}

William R. Ledoux ${ }^{2}$

VA Center of Excellence for Limb Loss Prevention and Prosthetic Engineering, Seattle, WA 98108;

Department of Orthopaedics \& Sports Medicine,

University of Washington,

Seattle, WA 98195;

Department of Mechanical Engineering, University of Washington,

Seattle, WA 98195

e-mail:wrledoux@u.washington.edu

\section{Michael Fassbind}

Eric S. Rohr

VA Center of Excellence for Limb Loss Prevention and Prosthetic Engineering, Seattle, WA 98108

Bruce J. Sangeorzan VA Center of Excellence for Limb Loss Prevention and Prosthetic Engineering, Seattle, WA 98108;

Department of Orthopaedics \& Sports Medicine, University of Washington,

Seattle, WA 98195

David Haynor
Department of Bioengineering,
University of Washington,
Seattle, WA 98195;
Department of Radiology,
University of Washington,
Seattle, WA 98195

\section{Multi-Rigid Image Segmentation and Registration for the Analysis of Joint Motion From Three-Dimensional Magnetic Resonance Imaging}

We report an image segmentation and registration method for studying joint morphology and kinematics from in vivo magnetic resonance imaging (MRI) scans and its application to the analysis of foot and ankle joint motion. Using an MRI-compatible positioning device, a foot was scanned in a single neutral and seven other positions ranging from maximum plantar flexion, inversion, and internal rotation to maximum dorsiflexion, eversion, and external rotation. A segmentation method combining graph cuts and level set was developed. In the subsequent registration step, a separate rigid body transformation for each bone was obtained by registering the neutral position dataset to each of the other ones, which produced an accurate description of the motion between them. The segmentation algorithm allowed a user to interactively delineate 14 foot bones in the neutral position volume in less than 30 min total (user and computer processing unit [CPU]) time. Registration to the seven other positions took approximately 10 additional minutes of user time and $5.25 \mathrm{~h}$ of CPU time. For validation, our results were compared with those obtained from 3DViewnix, a semiautomatic segmentation program. We achieved excellent agreement, with volume overlap ratios greater than $88 \%$ for all bones excluding the intermediate cuneiform and the lesser metatarsals. For the registration of the neutral scan to the seven other positions, the average overlap ratio is $94.25 \%$, while the minimum overlap ratio is $89.49 \%$ for the tibia between the neutral position and position 1, which might be due to different fields of view (FOV). To process a single foot in eight positions, our tool requires only minimal user interaction time (less than $30 \mathrm{~min}$ total), a level of improvement that has the potential to make joint motion analysis from MRI practical in research and clinical applications. [DOI: 10.1115/1.4005175]

Keywords: joint motion, MRI, segmentation, registration, visualization

\section{Introduction}

Accurate quantization of joint kinematics is a challenging task, especially for small complex joints such as those found in the foot and ankle. (While the examples discussed in this paper are foot specific, the principles apply to any joint in the body.) Methods include $\mathrm{X}$-ray stereophotogrammetry $[1,2]$, time sequence magnetic resonance imaging (MRI) [3], retro-reflective markers, placed either on the skin [4] or on bone pins [5], or recently, single [6] and biplane fluoroscopy $[7,8]$. Some of these methods involve ionizing radiation

${ }^{1}$ Present address: Biomedical Genomics Core, the Research Institute at Nationwide Children's Hospital, Columbus, OH 43205.

${ }^{2}$ Corresponding author. VA Puget Sound, MS 151, 1660 S. Columbian Way, Seattle WA 98108.

Contributed by the Biomechanical Engineering Division of ASME for publication in the Journal of Biomechanical EngineERING. Manuscript received June 20, 2011; final manuscript received August 29, 2011; published online October 31, 2011. Editor: Michael Sacks.

This material is declared a work of the US Government and is not subject to copyright protection in the United States. Approved for public release; distribution is unlimited.
(X-ray stereophotogrammetry and fluoroscopy) or are highly invasive (X-ray stereophotogrammetry and bone pins), leaving time sequence MRI, and skin mounted markers as the safest methods. In some clinical applications where the range of motion and the axes of rotation are of primary interest, a joint motion study using MRI has advantages over tracking techniques using surface skin markers: it is noninvasive, more accurate, and free from the effects of the sliding and deformation of skin and soft tissue [9]. MRI also produces a detailed depiction of joint anatomy. The principal disadvantages are the relatively low frame rate and the challenge of reproducing the effects of weight-bearing in a horizontal bore imager. An additional bottleneck exists in the processing of the acquired images; it is this aspect that our study has addressed.

Fully automatic methods, which can accurately segment each bone, are difficult to develop, while manual segmentation is a tedious procedure that also suffers from inter-observer variation and low reproducibility. For example, segmentation of the foot and ankle bones with the Live Wire method [10] requires $3 \mathrm{~h}$ for each scan, while using techniques based on National Institutes of 
Health (NIH) Image can require up to $10 \mathrm{~h}$ per scan [11]. (Note that all time estimates in this paper are based upon work in our laboratory.) A technique that would significantly reduce manual input while achieving comparable results is highly desirable.

Some of the methods, such as 3DViewnix, use principal component axes to determine the motion parameters [10], so an additional segmentation must be performed for each position. This makes the user operation time proportional to the number of positions. Principal component axes-based methods also have other drawbacks: (1) principal axes are ill-defined for bones with high symmetry; (2) the computation of principal axes is sensitive to segmentation result; (3) much information is lost by converting volume intensity to three principal component axes; (4) the truncation of long bones (e.g., the tibia, as often necessary when scanning the foot) has to be treated specially to get consistent principal component axes. Methods such as in Ref. [12] have been proposed to address this last issue. Finally, using principal axes for individual bones can lead to bone-to-bone relationships that were not clinically meaningful and difficult to describe in the cardinal planes [11]. By contrast, in the last 15 years voxel intensity-based medical image registration techniques have been widely used and validated in many areas, and it has become routine to achieve sub-voxel accuracy in image registration [13].

The purpose of this study is to describe and validate MultiRigid, an image segmentation and registration tool that employs rapid segmentation and automated image registration for studying joint motion. It allows for high quality segmentation of 14 bones of the foot in less than $30 \mathrm{~min}$ (user plus computer processing unit [CPU] time) and uses intensity-based image registration to solve for the motion parameters so only the images in one position need to be segmented no matter how many other positions are involved. In our experience with a dataset with eight positions and no automated registration, previous methods such as 3DViewnix would require 24-32 h of manual segmentation, while NIH Image-based methods would require on the order of $80 \mathrm{~h}$ of manual segmentation. However, the new method was able to process all 8 positions in less than $6 \mathrm{~h}$ (including less than 30 min total user interaction). The results were validated by comparison to existing methods and also by demonstrating a high degree of self-consistency.

\section{Methods}

Experimental Image Acquisition. MRI scans of a foot were performed using a custom designed positioning and loading apparatus that was based on the ankle loading device (ALD) [3]. Eight sets of three-dimensional (3D) images of the foot were obtained on a 1.5 T Philips MRI scanner (Philips Medical Systems, N.A.; Bothell WA), with the foot in the neutral position and seven other positions ranging from maximum plantar flexion, inversion, and internal rotation to maximum dorsiflexion, eversion, and external rotation. $3 \mathrm{D}$ sagittal $\mathrm{T}_{1}$-weighted images were acquired with an inplane resolution and slice thickness of $0.6 \mathrm{~mm} \times 0.6 \mathrm{~mm} \times 0.6 \mathrm{~mm}$ for the neutral position and $0.6 \mathrm{~mm} \times 0.6 \mathrm{~mm} \times 1.4 \mathrm{~mm}$ for the other positions, respectively. The MRI scan time was about 5 min for the neutral scan and 3 min for each of the other scans. The datasets were resampled to volumes with isometric voxels of $0.6 \mathrm{~mm}$ $\times 0.6 \mathrm{~mm} \times 0.6 \mathrm{~mm}$.

Segmentation. We developed a generic user interface that could be customized to specific anatomies for semiautomatic delineation of multiple bones (Fig. 1) that combines graph cuts [14] and level set $[15,16]$. Graph cuts is used interactively to obtain an initial segmentation, which is then automatically refined with the level set method. We used the foot/ankle interface to delineate 14 bones in the baseline (neutral) scan: tibia, fibula, talus, calcaneus, navicular, cuboid, medial cuneiform, intermediate cuneiform, lateral cuneiform, and the first through fifth metatarsals.

Graph Cuts for Image Segmentation. To apply the technique of graph cuts [14] to image segmentation problems, a graph is created in which each voxel in the image is represented by a node. Two additional distinguished nodes are introduced, representing the foreground object (the source) and the set of nonforeground voxels (the sink). Edges are introduced between neighboring voxels (the $n$-links) and between each voxel and the two special nodes (the $t$-links). Appropriate weights are chosen, which are small for edges connecting nodes with large intensity difference and large for edges connecting nodes with similar intensity. A minimum-cost cut that separates the two special nodes may then be shown to represent a good partition of the volume into the object and the background. Finding such a cut is a combinatorial optimization problem that has been extensively studied. When there are only two terminal nodes and when some restrictions on graph topology and the cost functions are satisfied, algorithms exist that can efficiently find the global minimum in polynomial time [17]. Modifications of these algorithms can be used to find good, although suboptimal, solutions to the multiobject segmentation problem.

We follow the method of Boykov for setting up the edge weights [18]. The weight assigned to the $n$-links between voxels $p$ and $q$ is

$$
w(p, q)=\exp \left(-d^{2} / \sigma_{n}^{2}\right)
$$

where $d$ is the gradient magnitude at the middle point between $p$ and $q$, and $\sigma_{n}$ is a parameter that controls the degree of smoothing. If $p$ and $q$ are at an object-background boundary, the gradient is large and this weight is small, favoring a cut between $p$ and $q$. For the object seeds, the weights for their $t$-links to the source and sink are set to $+\infty$ and zero, respectively, and vice versa for the background seeds. Because the image intensity of bone is not unique on MRI (e.g., the fat can be as bright as the trabecular bone), we set the $t$-link weights to zero for nonseed voxels. Finding the minimum cost cut for the graph structure above partitions the volume into two disjoint regions. To segment out multiple bones we run this binary segmentation sequentially, each iteration finding one bone while treating the rest of the image volume as the background. This is achieved by simply modifying the $t$-links: in each iteration we reassign the $t$-links while keeping the topology of the graph and the $n$-links unchanged.

Using a multiplanar viewer (Fig. 1), the user begins by rapidly indicating the seed voxels for each bone, which are treated as hard constraints. Axial, coronal, and sagittal plane slices are shown concurrently and synchronized by the position of the cursor. The user can draw the seeds for any bone on any slice and in any plane, and the other slices will be updated to reflect the seeds. After selecting seeds for all bones and the background, graph cuts are repeatedly applied for each bone. In each iteration only the seeds for current bone are regarded as the object and the seeds for the other bones are combined with the seeds for the background. The user can add/change the seeds if the segmentation results are not satisfactory. While in this scheme the final segmentation results might depend on the order of bones in which they are processed, we find the difference to be negligible in practice. For an operator familiar with the anatomy of the foot, the user interaction time is usually less than a minute to segment each bone.

Level Set for Segmentation Refinement. Graph cuts segmentation method is efficient and allows the user to arrive at an acceptable segmentation result quickly (Fig. 2(a)). It also has the capability to let the user interactively refine the result by modifying the seeds. However, it suffers from a lack of higher order smoothness constraints (Figs. 2(a) and 2(c)). Although Boykov and Kolmogorov [19] established a connection between graph cuts and continuous geodesic geometry and proposed a way of assigning the weights for $n$-links to simulate higher order smoothness constraints, this method is basically infeasible in practice, as too many additional edges are required.

To improve the segmentation result, we use a level set method after graph cuts. For each bone, the fast marching method [16] is run to convert the region label obtained from graph cuts to an 


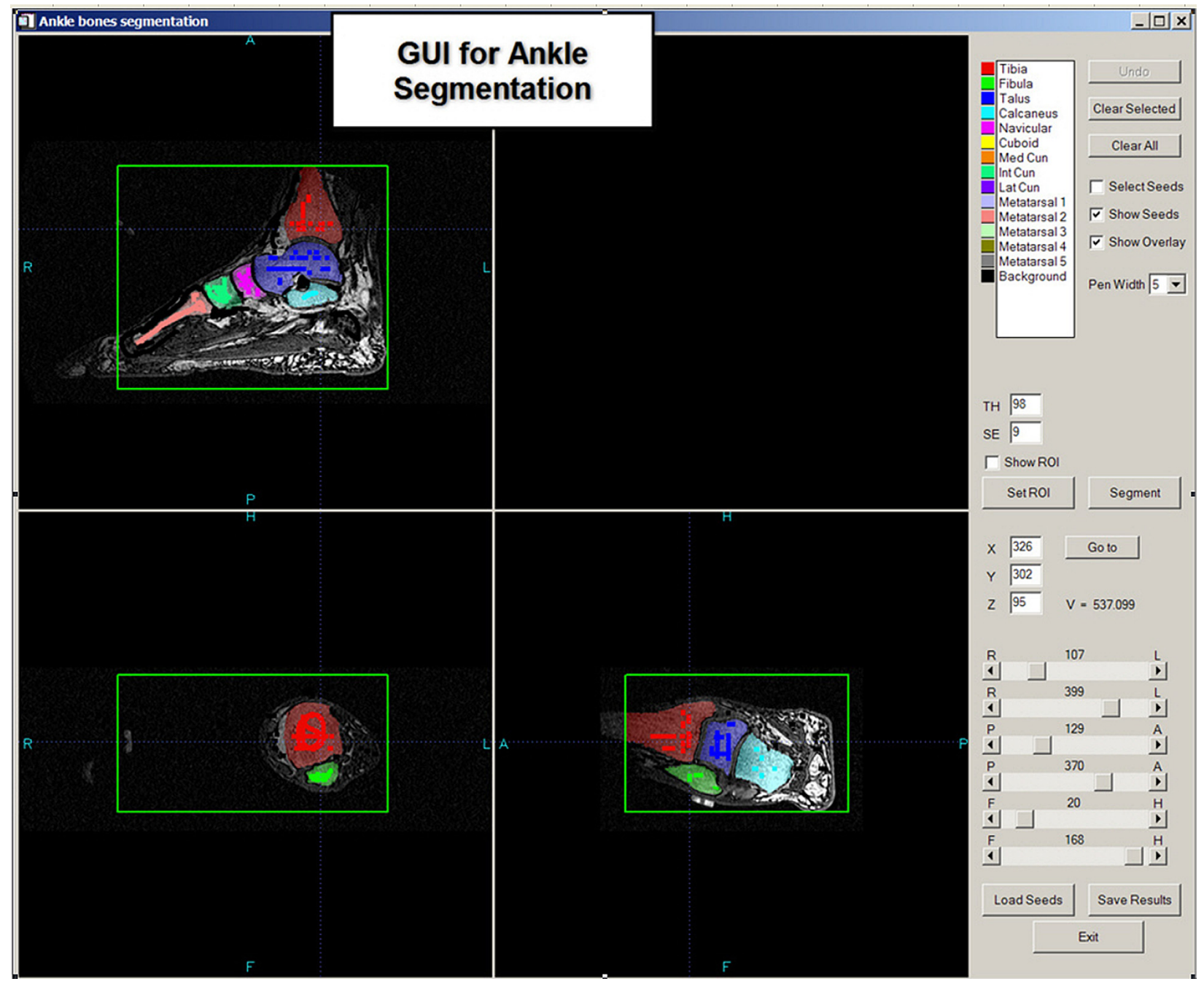

Fig. 1 Segmentation graphic user interface (GUI). Three orthogonal slices through the volume are displayed, on each of which the user can draw strokes freely to mark and segment different bones (shown in different colors). The segmentation results are also represented as color overlays on the images.

initial distance function, which is then taken as the starting input to the level set module. The speed function for the fast marching method is constant everywhere to obtain an approximate distance map. The speed function for the level set module follows the choice described by $\mathrm{Hu}$ and Haynor [20]

$$
F_{0}(\vec{x})=\omega_{1} \cdot S\left(\left|\nabla G_{\sigma}[I(\vec{x})]\right|\right) \cdot S(I(\vec{x}))+\omega_{2} \cdot \kappa
$$

which is the weighted sum of an image-based term $\left(S\left(\mid \nabla G_{\sigma}\right.\right.$ $[I(\vec{x})] \mid) \cdot S(I(\vec{x})))$ and a curvature term $(\kappa)$. The image-based term is the product of two sigmoid functions: the first one is a soft threshold on the Gaussian gradient magnitude $\left(\left|\nabla G_{\sigma}[I(\vec{x})]\right|\right)$ with $\sigma$ being the Gaussian kernel standard deviation, and the second one is a soft threshold on the image intensity. The parameters are chosen such that the speed function is large in regions with bone intensity and low gradient, but small in regions with nonbone intensity or high gradient. In choosing the soft threshold function, we use the sigmoid functions of the form

$$
S(I)=1 /(1+\exp (-(I-\beta) / \alpha))
$$

where the parameters $\alpha$ and $\beta$ are chosen empirically, for each of the two sigmoid functions. In our implementation, the weights $\left(\omega_{1}\right.$ and $\left.\omega_{2}\right)$ are also chosen empirically.

We run the level set refinement for each bone separately. The labels of other bones in the results of graph cuts are used as a "forbidden region" (speed $=0$ ) when the contour of one bone is evolving, in order to prevent the final contours of bones from overlapping one another. Since the result from graph cuts is usually quite good, we limit the number of level set iterations to 30 . The segmentation results from graph cuts can be compared before and after level set refinement (Figs. 2(a), 2(b), 2(c) and 2(d)). It can be seen that the addition of curvature constraint makes the final result smoother and more accurate. The importance of refined segmentation obtained with the level set method is primarily in visualization; it does not significantly affect the estimates of bony motion, that is to say, that the registration process does not require the level set refinement. This is partly due to the fact that we add a "cuff" to the bony region in the motion estimation process, as will be described in the next section.

Registration. The segmentation step separates a single set of scanned images (i.e., neutral position) into a collection of individual bones. Rigid body registration can then be used for each bone separately to follow its individual motion across multiple scans. The registration process involves finding spatial correspondence for the bones in different scans, and the resultant transformation parameters describe their motion between these positions. Mutual information maximization [21] is used to estimate the transformation parameters. Although correlation coefficients can also be used for intra-modality registration, we found mutual information 


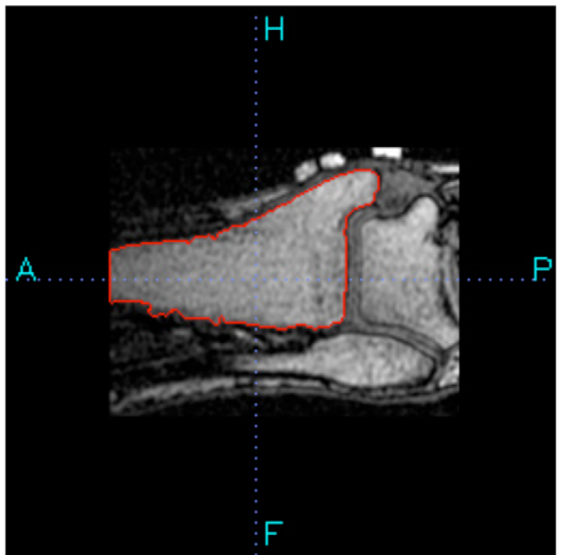

(a)

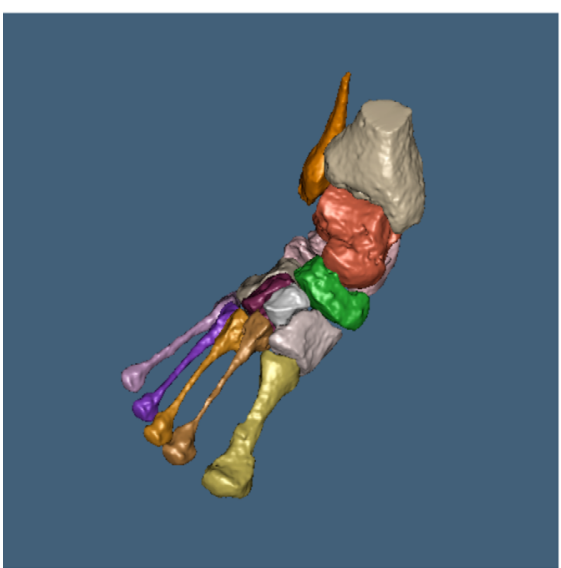

(c)

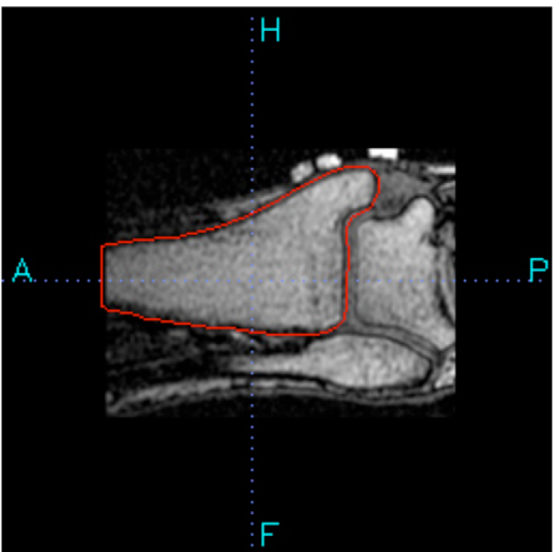

(b)

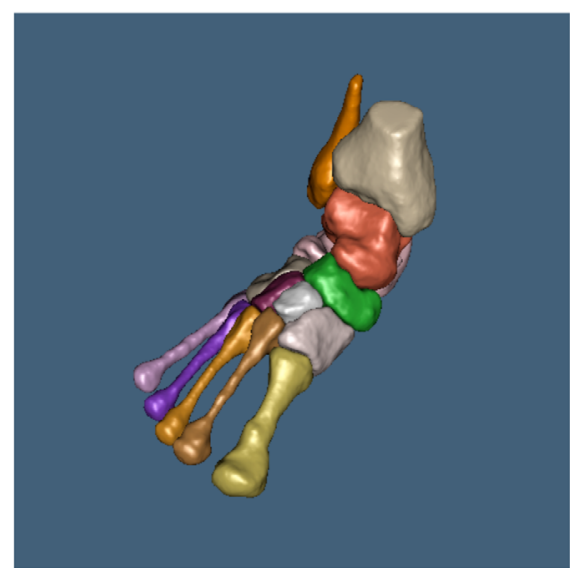

(d)

Fig. 2 Initial and final segmentation. (a): 2D results from graph cuts. (b): 2D results after level set refinement. (c): 3D results from graph cuts. (d): 3D results after level set refinement.

more robust. A Parzen window was used to deal with the discrete quantization problem associated with 2D histogram binning for probability distribution estimation. The transformations are parameterized with three Cardan angles and three translations. Cubic B-splines were used for image interpolation [22], and Powell's method [23] was used for optimization. The starting point for registration is two roughly corresponding points that the user picks manually, one for each scan or position scan. This process usually requires less than a minute of user interaction time for each additional scan. Of the 14 foot bones segmented, only three (the tibia, talus, and calcaneus) need to have corresponding points selected; the initial position of the remaining foot bones is estimated from these three. We expect the transformation parameters difference between adjacent bones to be small so just one point pair per data set is sufficient to initialize the chain of registrations.

When registering each bone, mutual information is only computed within the region assigned by the initial segmentation to that bone. Because this region is usually much smaller than the entire volume, the whole registration process can be performed much faster. Since mutual information measures region homogeneity relationships between two images, the edge information on both images is important for the metric to accurately represent the goodness of alignment. To be certain that the edges are included, the segmented volumes are dilated using mathematical morphology to include a "cuff" of the surrounding soft tissue.

Validation. Validation of the results of 3D segmentation and registration is notoriously difficult because of the unavailability of a gold standard. We used the following methods of validation.
Segmentation. First, we compared the label of each segmented bone with a semiautomatic segmented result using Live Wire [10] in 3DViewnix. The volume overlap ratio is computed as

$$
\mathrm{R}=2 \mathrm{~V}_{\mathrm{A} \cap \mathrm{B}} /\left(\mathrm{V}_{\mathrm{A}}+\mathrm{V}_{\mathrm{B}}\right)
$$

where $V_{A}, V_{B}$ are the volumes from our segmentation result and Live Wire, respectively, and $\mathrm{V}_{\mathrm{A} \cap \mathrm{B}}$ is the overlapping volume. One foot was processed in the neutral position using both our segmentation method (i.e., Multi-Rigid) and Live Wire, and the volume overlap ratio for each bone was computed.

Registration. Due to the lack of a gold standard, we cannot directly measure the true registration accuracy; instead the registration results are validated by visual inspection and selfconsistency checks. The registration results are used to transform each bone from the neutral position to the other positions to generate a transformed volume, which is compared with the raw volume in the other position. A linked-cursor viewer and a checkerboard viewer are used for the user to visually compare the correspondence. All datasets were visually inspected after the registration was computed, and all were found to be visually satisfactory.

Another way to evaluate the accuracy of registration is to check the consistency of registration circuits [24], i.e., if we register image $I_{1}$ to $I_{2}, I_{2}$ to $I_{3}$, and $I_{1}$ to $I_{3}$ independently, the result of registering $I_{1}$ to $I_{3}$ should be close to the combined results of registering $I_{1}$ to $I_{2}$ and $I_{2}$ to $I_{3}$. Even though this equality almost never perfectly holds due to the presence of errors, the 
discrepancies can be used as a lower bound for registration accuracy. In our experiment, we used the transformed bone labels from neutral position to perform all pair wise registrations.

For a certain bone assume $\mathrm{T}_{12}$, a $4 \times 4$ homogeneous matrix, represents the transformation from position 1 to 2 , and that $T_{23}$ and $\mathrm{T}_{31}$ are defined analogously. A point $\mathrm{P}$ in the bone will have a new location $\mathrm{Q}$ after going around the registration circuit $I_{1} \rightarrow I_{2} \rightarrow I_{1}: Q=T_{31} T_{23} T_{12} P$. The discrepancy for point $P$ is then computed by

$$
\mathrm{D}=\mathrm{P}-\mathrm{Q}=\left(\mathrm{I}-\mathrm{T}_{31} \mathrm{~T}_{23} \mathrm{~T}_{12}\right) \cdot \mathrm{P}
$$

where I is the identity matrix. We carried out the registration circuit studies on one foot, for which we chose the neutral position and three other positions including the two extreme positions, so there were 24 possible combinations of registration circuits. The maximum, mean, and root means square (RMS) discrepancies over all combinations were computed for each bone.

Overall System. Finally, the overall results of segmentation and registration combined were validated by doing segmentation in all of the other positions for a single cadaver foot, and then comparing the volume overlap ratio (Eq. (2)) between the real segmentation and the data transformed from the neutral position using the registration results.

\section{Results}

A 3D surface rendering of a single cadaver foot demonstrated the results of the graph cuts and level set algorithms (Fig. 2). Initially, the user spent less than 10 min selecting the seeds for all 14 bones, and it took about three minutes to run the graph cuts segmentation on a PC with a Pentium- $42.6 \mathrm{GHz}$ CPU and $2 \mathrm{~GB}$ RAM. After that, the user added a few seeds to a few bones, requiring two to three minutes, and ran the segmentation again, which took another three minutes. This process was repeated once more and the user was satisfied with the results (Figs. 2(a) and $2(b)$ ). The level set refinement was then run for 5 minutes of CPU time to get the final results (Figs. $2(c)$ and $2(d)$ ). The entire process took less than $30 \mathrm{~min}$, of which less than 15 involved user interaction.

The registrations from the neutral position to seven other positions were completed in about $5.25 \mathrm{~h}$ after user initialization of the registration, which took about 10 min of user interaction. The computed transformations were represented by three Cardan angles and three translations that can be converted into other types of motion description, such as helical axis $[25,26]$ or dual Euler angle [27]. Animations of the ankle motion between multiple positions were generated using spline interpolation of the transformation parameters. The registration results of all datasets were satisfactory by visual inspection (Fig. 3). The percentage volume overlap ratio for the comparison of our segmentation results with

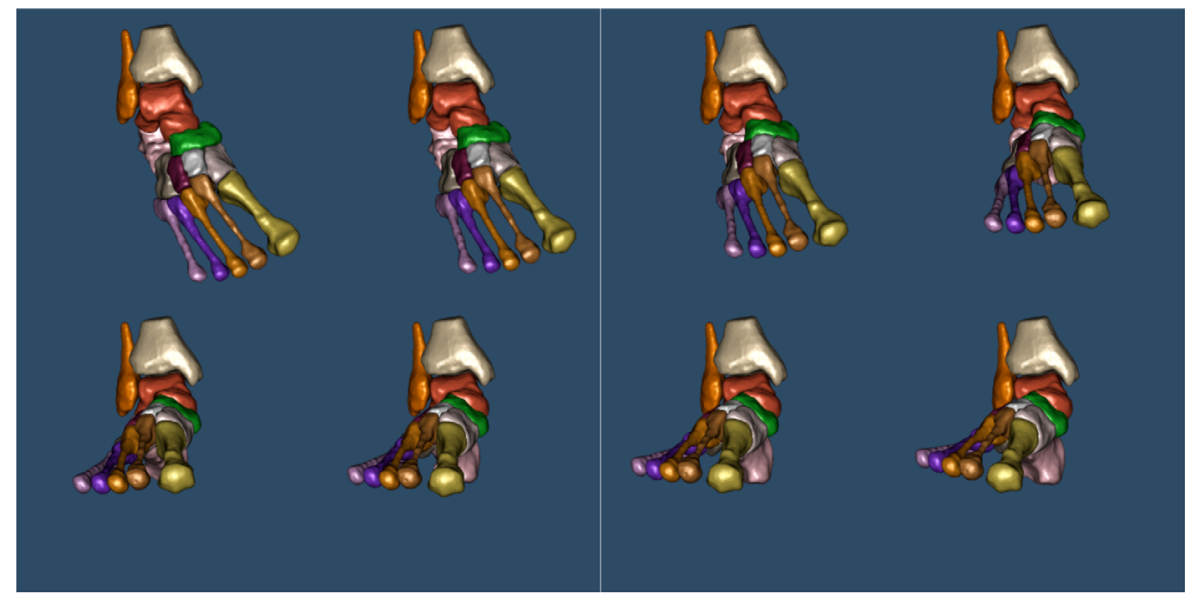

(a)

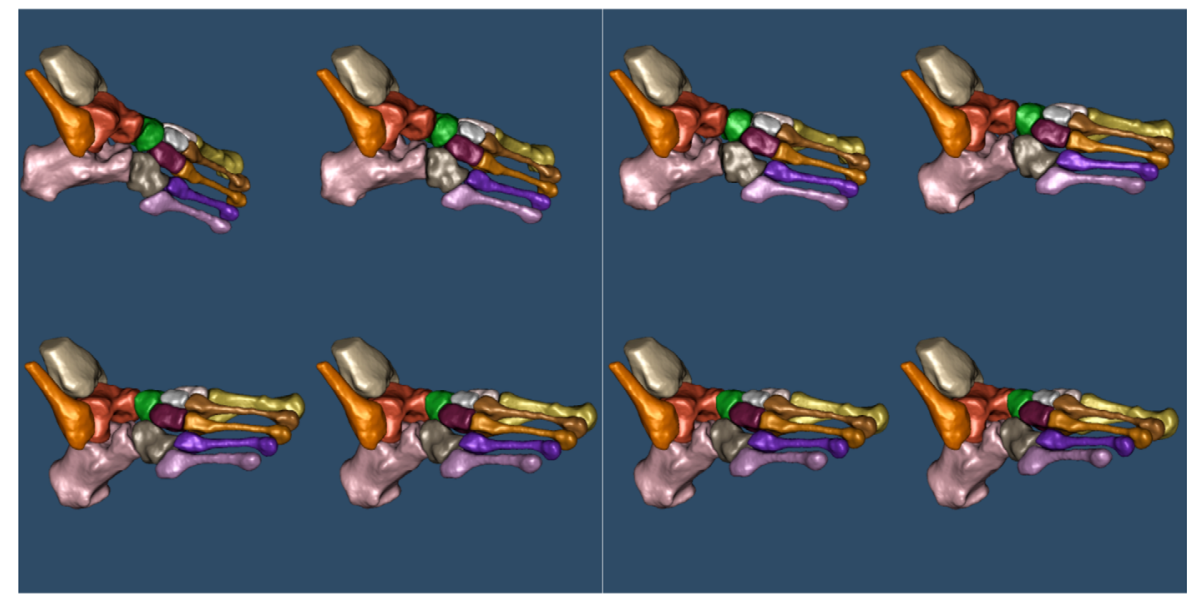

(b)

Fig. 3 Foot motion composite from eight MRI scans. (a) Top panel: anterior view, frontal plane; (b) bottom panel: lateral view, sagittal plane. From top left to bottom right: from plantar flexion, inversion, and internal rotation to dorsiflexion, eversion, and external rotation. In both panels, the upper right is the neutrally aligned scan. The tibia is fixed so all motions are relative to it. 
manual segmentation demonstrated good agreement, i.e., greater than $88 \%$ for all bones excluding the intermediate cuneiform and the lesser metatarsals (Table 1). Across all bones, the average volume overlap was $88.7 \pm 4.2 \%$; excluding the intermediate cuneiform and lesser metatarsals, it was $91.7 \pm 2.7 \%$.

As a further validation of the registration process, the maximum discrepancy for each bone over all possible combinations of registration circuits was less than $0.412 \mathrm{~mm}$ for all bones except the tibia and fibula (Table 1). The tibia and fibula have the largest discrepancy of around $0.5 \mathrm{~mm}$, partly due to that fact that these bones were truncated differently by the field of view (FOV) of different positions. Across all bones, the average maximum discrepancy was $0.296 \pm 0.131 \mathrm{~mm}$. The mean discrepancy for all bones except the tibia ranged from 0.038 to $0.120 \mathrm{~mm}$; the tibia mean discrepancy was $0.134 \mathrm{~mm}$. Across all bones, the average mean discrepancy was $0.087 \pm 0.031 \mathrm{~mm}$. The RMS discrepancy for all bones except the tibia ranged from 0.041 to $0.131 \mathrm{~mm}$; the tibia RMS discrepancy was $0.143 \mathrm{~mm}$. Across all bones, the average RMS discrepancy was $0.094 \pm 0.034 \mathrm{~mm}$.

The percentage volume overlap ratio for every bone between the transformed segmentation results from the neutral position and the segmentation results in every other position (i.e., a validation of segmentation and registration) was approximately 95\% (Table 2). The average overlap ratio is $94.25 \%$, while the minimum overlap ratio is $89.49 \%$ for the tibia between the neutral position and position 1, which might be due to different FOV. Considering that the errors from both segmentation and registration were cumulative in this measure, the agreement was very good.

\section{Discussion}

We have developed a method based on image segmentation and registration for studying joint morphology and motion and applied it to the complex anatomy of the foot and ankle, which comprises multiple bones and joints. We were able to process one subject's foot in eight positions in less than $6 \mathrm{~h} \mathrm{CPU}$ and user interaction time (30 min for segmentation and approximately $5.5 \mathrm{~h}$ for registration). The user interaction time was less than $30 \mathrm{~min}$ (approximately 15 min for segmentation and approximately 10 min for all registration initialization). This is a significant saving of time and manpower compared to the other methods, which required either three (3DViewnix) or ten (NIH Image) hours of user interaction time for each position. The accuracy of our method has been validated by extensive visual inspection, by comparison with an older,

Table 1 Volume overlap percentage (validation of segmentation) between Multi-Rigid and Live Wire for a single position (i.e., neutral). Discrepancy in $\mathrm{mm}$ (maximum, mean, and root mean square (RMS)) averaged over 24 combinations (validation of registration, see text for details).

\begin{tabular}{lcccc}
\hline \hline Bone & $\begin{array}{c}\text { Volume } \\
\text { overlap } \\
(\%)\end{array}$ & $\begin{array}{c}\text { Max } \\
\text { discrepancy } \\
(\mathrm{mm})\end{array}$ & $\begin{array}{c}\text { Mean } \\
\text { discrepancy } \\
(\mathrm{mm})\end{array}$ & $\begin{array}{c}\text { RMS } \\
\text { discrepancy } \\
(\mathrm{mm})\end{array}$ \\
\hline Tibia & 87.88 & 0.543 & 0.134 & 0.143 \\
Fibula & 89.39 & 0.513 & 0.118 & 0.127 \\
Talus & 94.39 & 0.327 & 0.120 & 0.128 \\
Calcaneus & 95.03 & 0.396 & 0.098 & 0.107 \\
Navicular & 92.57 & 0.255 & 0.091 & 0.098 \\
Cuboid & 93.21 & 0.149 & 0.048 & 0.050 \\
MedCun & 90.20 & 0.285 & 0.070 & 0.076 \\
IntCun & 85.26 & 0.144 & 0.038 & 0.041 \\
LatCun & 88.90 & 0.141 & 0.046 & 0.049 \\
Met1 & 88.96 & 0.314 & 0.108 & 0.118 \\
Met2 & 83.38 & 0.412 & 0.119 & 0.131 \\
Met3 & 81.67 & 0.166 & 0.061 & 0.067 \\
Met4 & 85.91 & 0.224 & 0.073 & 0.079 \\
Met5 & 84.61 & 0.272 & 0.095 & 0.103 \\
\hline \hline
\end{tabular}

well-validated method, and by confirming the self-consistency of registration circuits, which showed a maximum discrepancy of $0.54 \mathrm{~mm}$ and a mean discrepancy of $0.13 \mathrm{~mm}$. A critical observation is that, in order to accurately characterize bone motion using the Multi-Rigid software, it is unnecessary to have precise segmentations of the bones. "Pretty good" segmentations yield values for the automatically determined motion parameters identical to those derived for "good" segmentations, since the registration is largely driven by the bone-soft tissue interface.

The study of the motion of foot bones has been accomplished by various technologies in the past. Historically, this entailed the physical manipulating of cadaveric bones to identify joint axes $[28,29]$. Dual plane X-rays of feet with implanted markers have been used to quantify foot bone motion during various foot movements [1,2]. More recently, bone pins and kinematic markers have been employed in either living subjects [5] or cadavers [30]. While all of these methods can produce valid results, they either require the use of cadaveric tissue or are considered highly invasive for living subjects. Externally mounted retro-reflective markers may also be used [4], but these methods are susceptible to skin-motion artifact. Because of these limitations, various imaging modalities, including computed tomography (CT) $[11,31]$, MRI $[3,32,33]$, and biplane fluoroscopy $[7,8]$ will likely play increasingly important roles in foot kinematic studies. As such, it is necessary to have robust and accurate software for segmentation and registration that minimizes user interaction time.

The main contribution of this work is the novel segmentation approach that employs the graph cuts and level set methods and finds a good balance point between user interaction and automatic processing. Graph cuts are a combinatorial optimization technique that is discrete in nature, with efficient algorithms available for finding global optima in polynomial time under certain conditions $[17,18]$. By contrast, the level set method works by solving partial deferential equations (PDEs) using finite difference and is capable of incorporating higher order smoothness constraints such as curvatures $[15,16]$. Combining them sequentially therefore follows a conceptually multiresolution strategy: using graph cuts to interactively obtain coarse segmentation results guided by the user's high level knowledge of the anatomy, and then fine tuning the results for better accuracy with level set. This combination also overcomes a serious drawback of using level set alone, because it tends to converge to local optima without good initialization [34]. Compared to the method of using extra edges to simulate the higher-order smoothness of level set [19], our 2-stage approach is more computationally manageable. Another advantage to using graph cuts and level set is that both methods work in 3D (and higher dimensions as well) internally, unlike software packages such as 3DViewnix and NIH Image, which process 3D volumes on a slice-by-slice basis. Working directly in 3D is an important feature when dealing with objects of irregular shape such as the bones of the foot.

To find the motion parameters among different positions, we used mutual information maximization, a commonly used intensity based image registration method that is able to achieve subvoxel accuracy [13]. It is superior to methods based on principal axes because of its robustness, insensitivity to segmentation results, and ability to handle different FOVs due to truncation of long bones. Currently the registration procedure requires one pair of roughly corresponding points for the whole volume and can be further automated by utilizing application specific information (e.g., the expected range of motion for a foot between the neutral position and the first position towards maximum plantar flexion, inversion, and internal rotation can be used to initialize the registration).

Our Multi-Rigid approach has several limitations. While the user interaction time is significantly reduced, it is not completely eliminated. In addition, the automated registration process is rather slow (about six hours for eight positions). Future work includes automatic seeding for segmentation by deformable 
Table 2 Volume overlap ratio comparison (\%) for each bone between the neutral (position 5) and every other position (i.e., a validation of segmentation and registration)

\begin{tabular}{|c|c|c|c|c|c|c|c|c|c|}
\hline & Pos 1 & Pos 2 & Pos 3 & Pos 4 & Pos 6 & Pos 7 & Pos 8 & Ave. & Min. \\
\hline Tibia & 89.49 & 92.32 & 93.76 & 94.75 & 93.69 & 96.11 & 95.65 & 93.68 & 89.49 \\
\hline Fibula & 91.79 & 92.84 & 92.91 & 93.86 & 93.25 & 93.81 & 93.98 & 93.21 & 91.79 \\
\hline Talus & 93.79 & 94.53 & 95.04 & 96.02 & 96.00 & 95.39 & 95.19 & 95.14 & 93.79 \\
\hline Calcaneus & 95.93 & 96.98 & 96.77 & 97.23 & 96.62 & 96.05 & 96.23 & 96.54 & 95.93 \\
\hline Navicular & 94.03 & 94.04 & 94.30 & 95.54 & 94.32 & 95.31 & 93.88 & 94.49 & 93.88 \\
\hline Cuboid & 94.74 & 95.71 & 96.47 & 96.54 & 96.10 & 95.33 & 95.17 & 95.72 & 94.74 \\
\hline MedCun & 95.29 & 96.27 & 96.21 & 96.70 & 95.78 & 96.09 & 95.35 & 95.95 & 95.29 \\
\hline IntCun & 91.20 & 91.53 & 91.46 & 90.73 & 91.08 & 91.69 & 90.87 & 91.22 & 90.73 \\
\hline LatCun & 96.55 & 96.23 & 95.39 & 96.57 & 96.15 & 96.10 & 95.38 & 96.05 & 95.38 \\
\hline Met1 & 96.65 & 97.16 & 97.05 & 96.86 & 96.54 & 97.00 & 96.45 & 96.81 & 96.45 \\
\hline Met2 & 93.18 & 92.11 & 91.93 & 93.45 & 93.28 & 92.21 & 92.98 & 92.73 & 91.93 \\
\hline Met3 & 94.04 & 93.25 & 93.27 & 94.17 & 94.56 & 93.41 & 93.96 & 93.81 & 93.25 \\
\hline Met4 & 92.94 & 93.82 & 94.35 & 94.16 & 93.67 & 94.11 & 93.94 & 93.86 & 92.94 \\
\hline Met5 & 89.89 & 90.02 & 90.94 & 91.26 & 90.03 & 90.29 & 89.52 & 90.28 & 89.52 \\
\hline Average & 93.54 & 94.06 & 94.28 & 94.85 & 94.36 & 94.49 & 94.18 & 94.25 & \\
\hline Minimum & 89.49 & 90.02 & 90.94 & 90.73 & 90.03 & 90.29 & 89.52 & & 89.49 \\
\hline
\end{tabular}

registration of anatomic atlas to patient scans, and improving registration speed by exploiting more sophisticated optimization methods and parallel implementation on multi-CPU/multi-Core systems and massively parallel architectures such as graphical processing units (GPUs). Finally, while we have described and validated a new method for segmentation and registering bones in reduced times, we acknowledge that we have not exhaustively compared our software to every commercially available package.

In summary, we have presented a method, which we call MultiRigid, for the segmentation and registration of CT or MRI data. The technique involves two steps: the graph cuts method followed by the level set method. As representative application, we processed scans of a single foot that required less than $6 \mathrm{~h}$ to process, of which less than $30 \mathrm{~min}$ involved user interaction. These methods could be applied to other bones and joints in the body.

\section{Acknowledgment}

This research was partially supported by the National Institute of Arthritis and Musculoskeletal and Skin Diseases under Grant 5P60AR048093-03, and by the Department of Veterans Affairs, Rehabilitation Research and Development Grant A2661C.

\section{Nomenclature}

$$
\begin{aligned}
3 \mathrm{D} & =\text { three-dimensional } \\
\mathrm{ALD} & =\text { ankle loading device } \\
\mathrm{CPU} & =\text { computer processing unit } \\
\mathrm{CT} & =\text { computed tomography } \\
d= & \text { the gradient magnitude at the middle point } \\
& \text { between } p \text { and } q \\
\mathrm{D} & =\text { discrepancy between a point on bone in two loca- } \\
& \text { tions }(\mathrm{P} \text { and } \mathrm{Q}) \\
F_{0}(\vec{x}) & =\text { the speed function } \\
\mathrm{FOV} & =\text { fields of view } \\
\mathrm{GPU} & =\text { graphical processing unit } \\
\mathrm{I} & =\text { the identity matrix } \\
\mathrm{I}_{i} & =\text { Image } \\
I(\vec{x}) & =\text { image intensity } \\
\mathrm{MRI} & =\text { magnetic resonance imaging } \\
\mathrm{n}-\mathrm{links} & =\text { links between neighboring voxels } \\
p & =\text { representative voxel } \\
\mathrm{P} & =\text { a point on a bone } \\
q & =\text { representative voxel } \\
\mathrm{Q} & =\text { a new location for a point on a bone } \\
\mathrm{R} & =\text { overlap ratio } \\
\mathrm{RMS} & =\text { root mean square } \\
S(x) & =\text { sigmoid function } \\
t \text {-links } & =\text { links to two special nodes }
\end{aligned}
$$

\footnotetext{
$\mathrm{T}=$ transformation matrix

$\mathrm{T}_{1}$-weighted $=$ a type of MRI scan

$\mathrm{V}_{\mathrm{A}}=$ volume of Multi-Rigid segmentation

$\mathrm{V}_{\mathrm{B}}=$ volume of Live Wire segmentation

$V_{A \cap B}=$ overlapping volume

$\alpha=$ empirical parameter

$\beta=$ empirical parameter

$\left|\nabla G_{\sigma}\right|=$ the Gaussian gradient magnitude

$\kappa=$ curvature term

$\sigma=$ the Gaussian kernel standard deviation

$\sigma_{n}=$ a parameter that controls the degree of smoothing

$\omega_{1}$ and $\omega_{2}=$ weighting factors
}

\section{References}

[1] Lundberg, A., 1989, "Kinematics of the Ankle and Foot. In Vivo Roentgen Stereophotogrammetry," Acta Orthop. Scand. Suppl., 233, pp. 1-24.

[2] Van Langelaan, E. J., 1983, "A Kinematic Analysis of the Tarsal Joints, an X-Ray Photogrammetric Study,” Acta Orthop. Scand. Suppl., 204, pp. 1-269.

[3] Siegler, S., Udupa, J. K., Ringleb, S. I., Imhauser, C. W., Hirsch, B. E., Odhner, D., Saha, P. K., Okereke, E., and Roach, N., 2005, "Mechanics of the Ankle and Subtalar Joints Revealed through a 3D Quasi-Static Stress MRI Technique," J. Biomech., 38(3), pp. 567-78.

[4] Khazzam, M., Long, J. T., Marks, R. M., and Harris, G. F., 2007, "Kinematic Changes of the Foot and Ankle in Patients with Systemic Rheumatoid Arthritis and Forefoot Deformity," J. Orthop. Res., 25(3), pp. 319-29.

[5] Lundgren, P., Nester, C., Liu, A., Arndt, A., Jones, R., Stacoff, A., Wolf, P., and Lundberg, A., 2008, "Invasive in Vivo Measurement of Rear-, Mid- and Forefoot Motion During Walking," Gait Posture, 28(1), pp. 93-100.

[6] Yamaguchi, S., Sasho, T., Kato, H., Kuroyanagi, Y., and Banks, S. A., 2009, "Ankle and Subtalar Kinematics During Dorsiflexion-Plantarflexion Activities," Foot Ankle Int., 30(4), pp. 361-6.

[7] De Asla, R. J., Wan, L., Rubash, H. E., and Li, G., 2006, "Six Dof in Vivo Kinematics of the Ankle Joint Complex: Application of a Combined DualOrthogonal Fluoroscopic and Magnetic Resonance Imaging Technique," J. Orthop. Res., 24(5), pp. 1019-27.

[8] Li, G., Wan, L., and Kozanek, M., 2008 "Determination of Real-Time in-Vivo Cartilage Contact Deformation in the Ankle Joint," J. Biomech., 41(1), pp. $128-36$.

[9] Reinschmidt, C., Van Den Bogert, A., Nigg, B., Lundberg, A., and Murphy, N., 1997 "Effect of Skin Movement on the Analysis of Skeletal Knee Joint Motion During Running," J. Biomech., 30(7), pp. 729-32.

[10] Udupa, J., Hirsch, B., Hillstrom, H., Bauer, G., and Kneeland, J., 1998 "Analysis of in Vivo 3-D Internal Kinematics of the Joints of the Foot," IEEE Trans. Biomed. Eng., 45(11), pp. 1387-96.

[11] Ledoux, W. R., Rohr, E. S., Ching, R. P., and Sangeorzan, B. J., 2006, "Effect of Foot Shape on the Three-Dimensional Position of Foot Bones," J. Orthop. Res., 24(12), pp. 2176-86.

[12] Saha, P., Udupa, J., Falcao, A., Hirsch, B., and Siegler, S., 2004, "Iso-Shaping Rigid Bodies for Estimating Their Motion from Image Sequences," IEEE Trans. Med. Imaging, 23(1), pp. 63-72.

[13] West, J., Fitzpatrick, J., Wang, M., Dawant, B., Maurer, C. J., Kessler, R., and Maciunas, R., 1999, "Retrospective Intermodality Registration Techniques for Images of the Head: Surface-Based Versus Volume-Based," IEEE Trans. Med. Imaging, 18(2), pp. 144-50.

[14] Boykov, Y., Lee, V. S., Rusinek, H., and Bansal, R, 2001, "Segmentation of Dynamic N-D Data Sets Via Graph Cuts Using Markov Models," Medical 
Image Computing and Computer-Assisted Intervention (MICCAI), LNCS 2208, pp. 1058-1066.

[15] Osher, S. and Fedkiw, R., 2003, "Level Set Methods and Dynamic Implicit Surfaces," Applied Mathematical Sciences, Springer, New York.

[16] Sethian, J. A., 1999, "Level Set Methods and Fast Marching Methods: Evolving Interfaces in Computational Geometry, Fluid Mechanics, Computer Vision, and Materials Science," Cambridge Monograph on Applied and Computational Mathematics, Cambridge University Press, Cambridge.

[17] Boykov, Y., and Kolmogorov, V., 2004, "An Experimental Comparison of Min-Cut/Max-Flow Algorithms for Energy Minimization in Vision," IEEE Trans. Pattern Analysis Machine Intelligence, 26(9), pp. 1124-1137.

[18] Boykov, Y., and Jolly, M.-P., 2001, eds., Interactive Graph Cuts for Optimal Boundary \& Region Segmentation of Objects in N-D Images, Proceedings of the Eighth IEEE International Conference on Computer Vision, Vancouver, BC, Canada, July 7-14, 2001, IEEE, Vol. I, pp. 105-112.

[19] Boykov, Y., and Kolmogorov, V., eds, 2003, Computing Geodesics and Minimal Surfaces Via Graph Cuts, Proceedings of the Ninth IEEE International Conference on Computer Vision, Nice, France, Oct. 13-16, 2003, IEEE, Vol. I, pp. 26-33.

[20] Hu, Y., and Haynor, D. R., 2004, "Multirigid Registration of Mr and Ct Images of the Cervical Spine," Proc. SPIE, 5370, pp. 1527-1538.

[21] Viola, P., and Wells, W. M., 1997, "Alignment by Maximization of Mutual Information,” Int. J. Comp. Vision, 24(2), pp. 137-154.

[22] Unser, M., 1999, "Splines: A Perfect Fit for Signal and Image Processing," IEEE Signal Process. Mag., 16(6), pp. 22-38.

[23] Press, W. H., Flannery, B. P., Teukolsky, S. A., and Vetterling, W. T., 1992, Numerical Recipes in C: The Art of Scientific Computing, Cambridge University Press, New York.

[24] Holden, M., Hill, D. L. G., Denton, E. R. E., Jarosz, J. M., Cox, T. C. S., Rohlfing, T., Goodey, J., and Hawkes, D. J., 2000, "Voxel Similarity Measures for
3-D Serial Mr Brain Image Registration,” IEEE Trans. Med. Imaging, 19(2), pp. 94-102.

[25] Kinzel, G., Hall, A. J., and Hillberry, B., 1972, "Measurement of the Total Motion between Two Body Segments. I. Analytical Development," J. Biomech., 5(1), pp. 93-105.

[26] Kinzel, G., Hillberry, B., Hall, A. J., Van Sickle, D., and Harvey, W., 1972, "Measurement of the Total Motion between Two Body Segments. Ii. Description of Application," J. Biomech., 5(3), pp. 283-293.

[27] Ying, N., and Kim, W., 2002, "Use of Dual Euler Angles to Quantify the Three-Dimensional Joint Motion and Its Application to the Ankle Joint Complex," J. Biomech., 35(12), pp. 1647-1657.

[28] Manter, J. T., 1941, "Movements of the Subtalar and Transverse Tarsal Joints," Anatom. Rec., 80(4), pp. 397-410.

[29] Elftman, H., 1960, "The Transverse Tarsal Joint and Its Control," Clin. Orthop., 16, pp. $41-46$.

[30] Whittaker, E. C., Aubin, P. M., and Ledoux, W. R., 2011, "Foot Bone Kinematics as Measured in a Cadaveric Robotic Gait Simulator," Gait Posture 33(4), pp. 645-650.

[31] Beimers, L. M., Tuijthof, G. J., Blankevoort, L., Jonges, R., Maas, M., and Van Dijk, C. N., 2008, "In-Vivo Range of Motion of the Subtalar Joint Using Computed Tomography," J. Biomech., 41(7), pp. 1390-1397.

[32] Sheehan, F. T., 2010, "The Instantaneous Helical Axis of the Subtalar and Talocrural Joints: A Non-Invasive in Vivo Dynamic Study,” J. Foot Ankle Res., 3, pp. 13.

[33] Wolf, P., Luechinger, R., Boesiger, P., Stuessi, E., and Stacoff, A., 2007, “A Mr Imaging Procedure to Measure Tarsal Bone Rotations," J. Biomech. Eng., 129(6), pp. 931-36.

[34] Bae, E. and Tai, X.-C., 2009, "Efficient Global Minimization for the Multiphase Chan-Vese Model of Image Segmentation," Proceedings of the 7th International Conference on Energy Minimization Methods in Computer Vision and Pattern Recognition, pp. 28-41. 